\title{
Publisher Correction: Redefining ecosystem multifunctionality
}

Peter Manning, Fons van der Plas, Santiago Soliveres, Eric Allan, Fernando T. Maestre, Georgina Mace, Mark J. Whittingham and Markus Fischer

Correction to: Nature Ecology \& Evolution https://doi.org/10.1038/s41559-017-0461-7, published online 16 February 2018.

In the version of this Perspective originally published, in the figure in Box 3 the middle panel of the top row was incorrectly labelled ' $50 \%$ threshold-plus'; it should have read ' $50 \%$ threshold'. This has now been corrected. 